\title{
A STUDY OF EMOTIONAL EXPRESSION IN
} DE MENTIA PRÆCOX*

\author{
BY J. W. DE BRUYN
}

(From the Michigan State Psychopathic Hospital, Ann Arbor, Mich.)

T $N$ view of the large place that has been given to the pathological states in the discussions of the JamesLange theory of the emotions, it seemed to us that it might be worth while to study the more obscure reactions of the insane who gave little or no sign of emotional response. Patients suffering from dementia praecox, for the most part of the catatonic type, were chosen for investigation. As will be seen from the case descriptions given below they were all in some degree deficient in emotional expression and probably in some degree lacking in the appreciation of emotional states.

The responses chosen for study were the respiratory and vaso-motor, although the records also gave opportunity of studying the gross changes in the pulse. The apparatus used was the finger plethysmograph with piston recorder and the Sumner pneumograph with Marey tambour.

The stimuli used were of sufficient variety to eliminate any special effects due to the sense worked with. The method of applying the stimulus is given where it is not sufficiently evident from the mention of the stimulus.

The experiments were made upon dementia praecox patients at the Michigan State Hospital, in Ann Arbor, and at the Michigan State Asylum, located at Kalamazoo. We note here extracts from the histories of the patients experimented upon.

Gr: Patient is able to answer questions, but often is noticed whispering to himself and assuming peculiar attitudes. Orientated as to time and place. No tremor. Has hallucinations of hearing, smell, taste, and sight. Memory is fair. Has delusions concerning poisoning and hypnotism. No echophrasia, echolalia, or stereotypedness.

*Communicated by Prof. W. B. Pillsbury. 
When I came to work with the patient I found he had become mute. He also seemed to recognize me and to understand what I had to say to him.

Ro: When admitted, his pulse was very rapid. Memory poor. Did not converse. Depressed, untidy, and indifferent. Very resistant. Later showed more mental activity, whistling popular songs which had become popular since he entered the institution. Later, again, became indifferent to his surroundings, showed mannerisms and often smiled without occasion, and whispered to himself. I found him to be very agreeable, recognizing me whenever I entered his ward. He gave an excellent pulse and was rarely if at all constricted.

Ho: When admitted, seemed impulsive and maniacal, and usually would not answer questions. Would stop and gaze at a spot on the wall. Later, showed mannerisms, disturbed orientation, auditory hallucinations, and stereotyped manner of answering questions. I found him to be very silly, laughing so that results were often spoiled.

McK: A case of manic-depressive insanity or dementia praecox, hebephrenic type. Seems to have had periods of depression alternating with periods of elation. Judgment good but somewhat overshadowed by depressed ideas. Has depressed hallucinations of vision and hearing. When I came to work with him I found that he would not talk to me and obeyed orders almost resistantly.

Ma: At entrance was irritable and would not do what was asked of him. Remained in whatever position he was placed. Hallucinations of sight and hearing. Emotional state variable, depressed, apprehensive, or indifferent. Sometimes catatonic, at other times resistant to being placed into position. Impulsive outbreaks attacking those about him. Hears voices from above and does little or nothing. Not entirely orientated as to time. His grasp of immediate and remote past is poor. Rambles in his conversation.

Le: Dementia praecox of catatonic type. Negativism physically and mentally. When asked to do anything the first impulse is to do it, then to do the contrary. Has some stereotyped movements; at regular intervals jumps up from chair, stands a few minutes and sits down. 
Mi: At time of admission could speak. Said he heard voices. Later became mute and sat all day with hands folded and seemed to pay no attention to surroundings. Occasionally smiled to himself. I found this man timid and fearful. Badly constricted except once or twice.

$\mathrm{Ha}$ : When admitted had a slow, unsteady gait. Hallucinations of hearing; many of his acts were in response to them. Mildly elated. Indifferent toward self and environment. Some cataleptic tendencies. Spends most of his time playing an imaginary piano and singing a tune over and over. Is a case of well-advanced dementia praecox. No interest in things about. Sing-song utterances difficult to understand. Defective orientation. I found him to be a typical case of stereotypedness. While I was working with him I never found him attempting to play the piano, but he was actively hallucinated, muttering to himself constantly. He was the most negative as well as the best case with which I had to deal.

$\mathrm{Ch}$ : Megalomaniac. Occasional hallucinations.

Co: Orientated for time and place. This man is fairly normal. I was able to have him figure problems.

Sp: Quite normal, but is affected by his inability to control his laughter.

For the sake of convenience in comparison we give the results in two summaries, first with reference to the effects of stimuli upon the different patients and then with reference to the different stimuli that were used.

Ro: The volume was decreased once (vaso-constriction) and once the response was doubtful when kerosene was dropped upon the skin of the neck. Deep pin pricks gave once a delayed, once an immediate fall in volume. A shrill whistle near the ear gave a negative result once, one irregular change, and once an immediate, once a delayed constriction. A tin pan dropped upon the floor once gave an irregularity, once no response, and once a fall in volume. Of the gustatory stimuli applied salt gave irregularity, cinnamon no effect except once a fall in volume, chocolate a fall in volume. Of the odors camphor gave a doubtful result, ammonia in two instances a fall. The changes in respiration were rather more definite. The two tactual stimuli gave shallower 
respiration in every (four) instance. The whistle checked respiration three times out of four, while the tin pan once gave a fall, once a rise, and once no change. Salt gave deepened respiration once, cinnamon once no change, and once a deepening, chocolate a doubtful effect. Camphor produced irregular breathing, while ammonia in each case diminished the amplitude of the respiratory movement, perfume no effect. In summary, then, we find that in eighteen experiments there was fall in volume ten times, no change four times, and a doubtful result or mere irregularity four times. Respiration became more shallow ten times, deeper three times, irregular twice, and was unchanged four times.

Mi: Perfume twice gave increased volume, twice negative results. Camphor, sugar, and ammonia gave no results. A pin prick (severe) failed to produce a fall in volume but once out of five times; kerosene twice failed of effect, and once gave a fall followed by a rise, pinching a rise. The whistle gave a fall once, a rise once, and three times no result; the tin pan produced constriction each time it was used. Respiration was diminished once out of four times by perfume; not at all by camphor, ammonia, or sugar; in each of five trials by pin pricks; by pinching each time; by kerosene once out of three times, by the whistle once out of five times, and the tin pan once out of two trials. There was deepened respiration once by the whistle and once after an interval of diminution by the kerosene.

Ho: Kerosene and the pinch gave fall of volume, perfume and the whistle a fall of volume, while all of the stimuli mentioned gave shallower respiration.

$\mathrm{Me}$ : Camphor, ammonia, the whistle, and the tin pan gave no result, while the pin prick gave constriction and diminished respiration.

$\mathrm{Gr}$ : Perfume gave three cases of constriction in volume, one doubtful case; ammonia three: one restriction, one negative, and one uncertain; the whistle, one dilation, one dilation followed by constriction, one negative, and one doubtful case; a pan dropped upon the floor gave no response three times and once a rise followed by a fall; a pin 
prick induced two rises in volume followed by a fall; cinnamon one constriction and one negative result; sugar a fall in two cases, camphor one rise and one uncertain case; kerosene one fall and one uncertain result; chocolate an irregularity. In respiration perfume gave one case each of diminution, of diminution followed by an increase, an uncertain result, and a negative one; ammonia induced two diminutions, one irregular change, one was doubtful, and once there was no change; the whistle induced a diminution twice, a diminution followed by a rise once, and one negative case; the pan no change four times; a pin prick once diminished, once diminished then increased the breathing; sugar and camphor each gave one negative result and one diminution. In all, out of twenty-six trials there was constriction nine times, dilation twice; dilation followed by constriction four times, constriction followed by dilation once, five uncertain and five negative cases.

Approximately the same stimuli were used for the other cases, so that for the remaining patients we may spare the reader the details and give the bare general summary.

Ha: Thirteen tests. Six cases of constriction in volume, three of dilation followed by constriction, one negative, and one doubtful case. In respiration six diminutions, one diminution followed by increase, one increase followed by decrease, two negative and one doubtful case.

$\mathrm{Sp}$ : (nearly normal). Twenty tests. In volume there were four cases of fall, four of rise, two of rise followed by a fall, four negative and two doubtful, in respiration six checkings, three instances of no effect and five of doubtful result.

Co.: Nine tests. Of these two gave constriction, one constriction following dilation, five no result, and one a doubtful response. In respiration there were six negative cases, one decrease and one irregularity.

Ch.: Six tests. Only one constriction of capillaries three negative and one doubtful result. In respiration four were negative and one doubtful.

McK: Five tests, three cases of constriction, two doubtful, and two cases of diminished respiration, one negative, and one uncertain response. 
Ma: Six tests. Two cases of restriction, three negative and one doubtful response. Respiration was diminished in five instances, the effect was in doubt twice, and once there was no response.

Of the eleven patients experimented upon only two would be regarded as at all obtuse in their responses, and these, $\mathrm{Co}$. and $\mathrm{Ch}$., were among the patients more slightly affected. It is probable that the small percentage of positive results with them was due to the small intensity of the stimuli employed or to other extraneous circumstances.

In final-summary, we find that olfactory stimuli gave the following results. First as to volume: Seventeen experiments resulted in no change, twelve in a fall, nine in uncertainty, one in irregularity, five in a rise, and two a rise follon ed by a fall during stimulation. Second as to respiration: Twelve times there was no change, twelve times diminution, five times uncertainty, six times irregularity, one rise and two rises followed by falls during stimulation. Auditory stimuli gave the following changes in volume: Sixteen times there was no change, eight times a fall, six times uncertainty, twice a rise, three times a rise followed by a fall during stimulation, and once a fall followed by a rise during stimulation. As regards respiration, there was no change nineteen times, five falls, one uncertain response, four irregularities, one rise, and one rise followed by a fall during stimulation. The results regarding gustatory stimuli were these: As to volume, six times no change, eight falls, two uncertain, one irregular, and one rise followed by a fall during stimulation. As regards respiration, there was no change, six times, three falls, three questionable, one irregular, three rises, and two falls followed by rises during stimulation. Tactual stimuli caused the following records. As to volume, five times no change, nine times a fall, once uncertainty, eight rises, and one fall followed by a rise during stimulation. As to respiration, there was no change twice, eleven falls, two uncertain, one rise followed by a fall during stimulation, and two shallower breathings.

These results show that the character of the stimulus makes little difference, at least in dementia praecox cases, with the likelihood of stimulation. If, however, I were to 
be compelled to choose between stimuli with which to obtain most noticeable results, I would choose such tactual stimuli as pinching or pin-pricking, for these seem to effect most easily the instinctive processes.

The results themselves are unambiguous. In one hundred and seventy-seven experiments made on patients who showed practically no sign of emotion as judged by the ordinary tests there were one hundred and twenty-one in which the circulation of respiration showed some response. It would seem that the unresponsiveness of dementia praecox extends to the voluntary muscles alone and is not at all marked so far as the more involuntary activities are concerned. The interpretation of the results is, however, by no means so certain. Could one be sure that the patients were without emotion as well as defective in emotional expression it would seem that we could have emotional expression in one important particular without an accompanying emotion. The evidence for or against the presence of the emotion is not at all definite. The introspection of a patient at the stage of mental dissolution presented by most of our patients is very unreliable. Indirect evidence might be drawn from the fact that the patients' hallucinations in an actively hallucinated condition often went on unchanged in character and degree by the stimulus. This would seem fairly conclusive evidence that the stimulus aroused no emotion, but would not serve to differentiate the case from sleep where there are vital responses without waking, or from states of dissociation, like hysteria, where consciousness attaches to selected operations only, in spite of definite physiological response to others.

In the necessary ignorance of the mental content of the emotions, our results would only be sufficient to show that a distinction must be made between the voluntary and involuntary accompaniment of an emotion. Voluntary expression may be lacking, involuntary be present. Probably, too, the involuntary reactions may take place without the presence of an emotion as a conscious state.

Some evidence might also be drawn from the results as evidence that the passivity of catatonia is the result of inhibition rather than any defect in capacity. Were some defect in the mechanism present one would expect that it would affect 
the involuntary and the voluntary alike. Were it the result of active inhibition one would expect what we find, that the voluntary processes alone would be checked while the others were beyond control.

We desire to express our sense of obligation to Dr. Barrett for suggesting the problem and putting at our disposal the resources of the Psychopathic Hospital, to Dr. Shepard for assistance, in setting up the apparatus, and in its manipulation during the first experiments; to Dr. Ince, and the other officials of the State Hospital at Kalamazoo for the opportunity to use their patients, and for access to case histories and diagnoses.

\section{HYPNOIDAL STATES - A CORRECTION}

In the last number of the Journal in a footnote to my article on the "Unconscious"(p. 337), after giving Dr. Boris Sidis's definition of the state which he terms "hypnoidal," by a clerical error I was made to say: "This seems to be the same state which Brewer and Freud originally termed hypnoidal." The last word "hypnoidal" should have read hypnoid. As it stands it does injustice to Dr. Sidis, who coined the term hypnoidal and applied it to what he regards as a "subwaking state." Brewer and Freud have used the term hypnoid, as others may have done, to define certain dissociated conditions and particularly certain hysterical states. Sidis's state is certainly not an hysterical state. What I had in mind was whether or not it can be identified with that condition which is generally regarded as light hypnosis, and in which a person equally well can be said to be "in full possession of his powers and still is more closely in touch with the dissociated experiences than he is otherwise in the full waking state." Whatever the answer may be Dr. Sidis was the first to apply the term hypnoidal to a state which he has carefully and extensively studied. 